Abstract

\title{
Analyzing Amyloid Beta Aggregates with a Combinatorial Fluorescent Molecular Sensor ${ }^{\dagger}$
}

\author{
Joydev Hatai, David Margulies * and Leila Motiei \\ Department of Organic Chemistry, Weizmann Institute of Science, Rehovot 7610001, Israel; \\ joydev.hatai@weizmann.ac.il (J.H.); leila.motiei@weizmann.ac.il (L.M.) \\ * Correpondence: david.margulies@weizmann.ac.il \\ + Presented at the 5th International Symposium on Sensor Science (I3S 2017), Barcelona, Spain, \\ 27-29 September 2017.
}

Published: 6 December 2017

The self-assembly of amyloid beta $(\mathrm{A} \beta)$ peptides into insoluble aggregates is thought to play a major role in the progression of various neurodegenerative diseases, including Alzheimer's disease (AD). Although various studies have shown that subtle variations in the dynamics and compositions of $A \beta$ aggregates could have a significant impact on their physicochemical and pathological properties, currently there is no effective means to straightforwardly characterize the $A \beta$ aggregation state. Fluorescent assays, which mainly rely on the 'turn-on' properties of a thioflavin T (ThT) molecule, can only detect the fibril formation, whereas other techniques that can determine the content of these assemblies require special expertise and are not high-throughput. To improve the ability to analyze $A \beta$ aggregates, we have developed a combinatorial fluorescent molecular sensor that generates a wide range of unique emission 'fingerprints' upon binding to distinct $A \beta$ aggregate species. The molecular sensor has been used to discriminate among aggregates generated from different alloforms (i.e., $A \beta_{40}$ and $A \beta_{42}$ ) or through distinct pathways, and it has also been used to track dynamic changes that occur in $A \beta$ aggregation states, which result from the formation of low molecular weight (LMW) oligomers, high molecular weight (HMW), oligomers, protofibrils, and fibrils. To demonstrate the versatility of our approach, we have also shown that the sensor can discriminate among aggregates of other amyloidogenic proteins, such as lysozyme, prion, insulin, and amylin fibrils (Published in J. Am. Chem. Soc. 2017, 139, 2136-2139. Highlighted in JACS Spotlights).

(C) 2017 by the authors. Licensee MDPI, Basel, Switzerland. This article is an open access article distributed under the terms and conditions of the Creative Commons Attribution (CC BY) license (http://creativecommons.org/licenses/by/4.0/). 